\title{
Consideration on Elastic Vibration Control of a Magnetically Levitated Thin Steel Plate Using Sliding Mode Control*
}

\author{
Shinya HASEGAWA**, Yasuo OSHINOYA*** and Kazuhisa ISHIBASHI ${ }^{* * * *}$
}

\begin{abstract}
We have proposed a magnetic levitation control system for a sheet steel and confirmed the realization by a digital control experiment. However, because of the strong nonlineality of the attractive force of the electromagnet and the various uncertainties in the circuit current such as changes in the resistance due to heat generation of the electromagnet, stability of levitation has not been sufficiently ensured. In this study, we aim to develop a noncontact support system for thin steel plates with high robustness using sliding mode control, which is tolerant to factors such as disturbances within control signals and external forces affecting the system. As a result, it was verified that the suppressive effect of the sliding mode control on disturbances is sufficient, and that the application of the continuous model provides the construction of a system with robustness to the disturbance of the external forces.
\end{abstract}

Key Words: Electromagnetic Actuator, Vibration Control, Optimal Control, Sliding Mode Control, Steel Plate, Electromagnetic Levitation, Elastic Vibration, Continuous Model and Disturbance

\section{Introduction}

Thin steel plates are widely used as materials for automobiles, electric appliances, cans and other products in current industries. With various industrial demands, the surface quality of steel plates continues to be enhanced. However, because contact conveyance using rollers is mainly adopted in the process of a thin-steel-plate production line, the problem of surface quality deterioration arises. In recent years, as a countermeasure for this problem, research on the noncontact conveyance system with the application of electromagnetic levitation technology has become active ${ }^{(1)-(3)}$. In the past, our research group has constructed an electromagnetic levitation control system with which the relative distance between an electromagnet and a steel plate is constantly maintained, aiming to prevent the steel plate from falling from the conveyer or

* Received 31st May, 2004 (No. 04-4137)

** Graduate School of Tokai University, 1117 Kitakaname, Hiratsuka-shi, Kanagawa 259-1292, Japan

*** Department of Mechanical Engineering, Faculty of Engineering, Tokai University, 1117 Kitakaname, Hiratsukashi, Kanagawa 259-1292, Japan.

E-mail: ossy@keyaki.cc.u-tokai.ac.jp

**** Department of Mechanical Engineering, Faculty of Engineering II, Tokai University, 2-28-4 Tomigaya, Shibuyaku, Tokyo 151-8677, Japan coming into contact with the electromagnet during electromagnetic levitation conveyance ${ }^{(4)}$. However, due to the strong nonlineality of the attractive force of the electromagnet and the various uncertainties in the circuit current, such as changes in the resistance due to heat generation of the electromagnet, the stability of levitation is not sufficiently ensured. As one of the effective control methods for solving this problem, sliding mode control, which enables easy handling of nonlinear models, is drawing attention, and an attempt to use it in electromagnetic bearing control has been reported ${ }^{(5),(6)}$. With its theoretical capability of completely removing disturbances and modeling errors existing in the same channel as the control input ${ }^{(7)}$, or any disturbance satisfying the matching condition, sliding mode control is highly robust. Accordingly, when sliding mode control is applied to electromagnetic levitation control of thin steel plates, it is expected not only to show excellent control performance against the deterioration of the levitational effect due to the nonlinearlity of the electromagnetic attractive force, but also to ensure robustness against various uncertainties in the circuit current which cannot be eliminated by applying a general linear control theory, thereby enabling the construction of more stable control systems. Under such circumstances, in this study, we simulated uncertainties in the circuit current by forcibly inputting disturbances into control signals of an electromagnet used for the levitation of thin steel plates, 
and investigated the effect of sliding mode control in suppressing such disturbances.

Another major problem in electromagnetic levitation control of thin steel plates is the lack of levitation stability due to elastic vibration of the plate, which is a flexible body. Assuming an actual electromagnetic levitation conveyance process, a thin steel plate is likely to undergo elastic vibration due to an unexpected external force other than the supporting electromagnets. However, no attempt has been reported regarding artificially creating elastic vibration and controlling it during the levitation of the plate. Therefore, we installed an electromagnet in order to produce disturbances in an experimental apparatus, and artificially applied an external force to the thin steel plate during levitation by electromagnetic attractive force, and investigated the effect of suppressing the elastic vibration. We also performed a comprehensive investigation wherein these disturbances were applied simultaneously, assuming an actual industrial process.

To model a thin steel plate, we applied two different models; a continuous model considering the vibration mode, and a distributed model with which a control system can be easily designed. Then, discussion was made from the viewpoint of a performance evaluation of the sliding mode applied to these two models. Moreover, in this study we designed a control system using discrete-time sliding mode control ${ }^{(8)}$. With the suppression of chattering, this system controls the spillover of higher order electric vibration modes, that had previously been neglected. In addition, an optimal control was also adopted to compare the control performance, and experiments and numerical simulations were performed for various cases.

\section{System for Control Experiment}

Figure 1 shows an outline of the control system and experimental apparatus ${ }^{(4)}$. The object of electromagnetic levitation is a rectangular zinc-coated steel plate (SS400) with length $a=800 \mathrm{~mm}$, width $b=600 \mathrm{~mm}$, and thickness $h=0.3 \mathrm{~mm}$. In order to accomplish noncontact support of the steel plate using 5 pairs of electromagnets (Nos. 1-5) as if the plate was hoisted by strings, the displacement of the steel plate is measured by five eddy-current gap sensors. The electric circuits of these paired electromagnets are connected in series, while an eddy-current gap sensor is positioned between the two magnets of each pair. Five displacements detected at each position of the electromagnets and five electric currents calculated from the external resistance are together input to DSP through the ten channels of an A/D converter. Velocity can also be calculated by means of digital differentiation of the displacement using the DSP. Thus obtained 15 values are used to calculate the control voltage over each of the five electromagnets. A control voltage is output from the five channels of the D/A converter into a current-supply amplifier to control the attractive force of the 5 pairs of electromagnets, realizing levitation of the plate below the surface of the electromagnets by $5 \mathrm{~mm}$. In this study, for the basic examinations, the steel plate is reinforced by three pipes in order to suppress the elastic vibrations in the $x$-axis direction. The dotted lines in the steel plate shown in Fig. 1 are the pipes made of light carbon fiber. The total weight of the carbon pipes is approximately $6 \%$ of the steel plate mass. It is confirmed that the influence of attaching a pipe to the steel is negligible when low-order modes, such as first- and second-order modes, are considered. In addition, as shown in Fig. 2, two electromagnets for the gen-

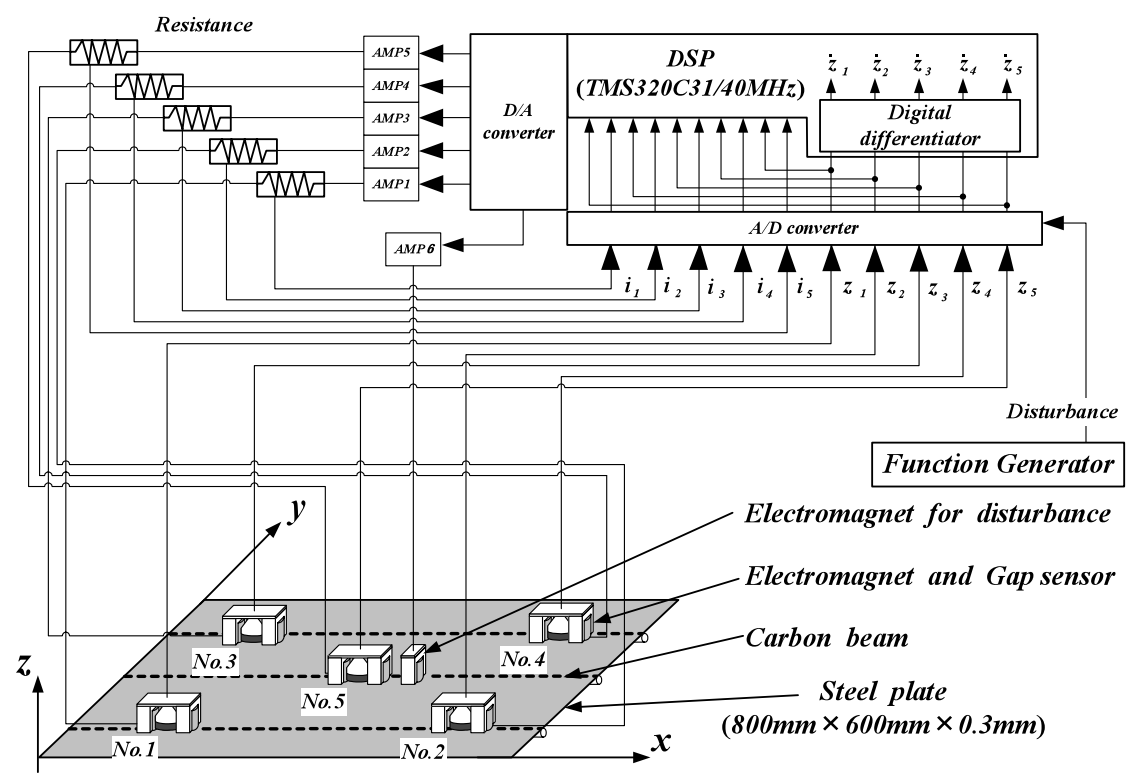

Fig. 1 Electromagnetic levitation control system 


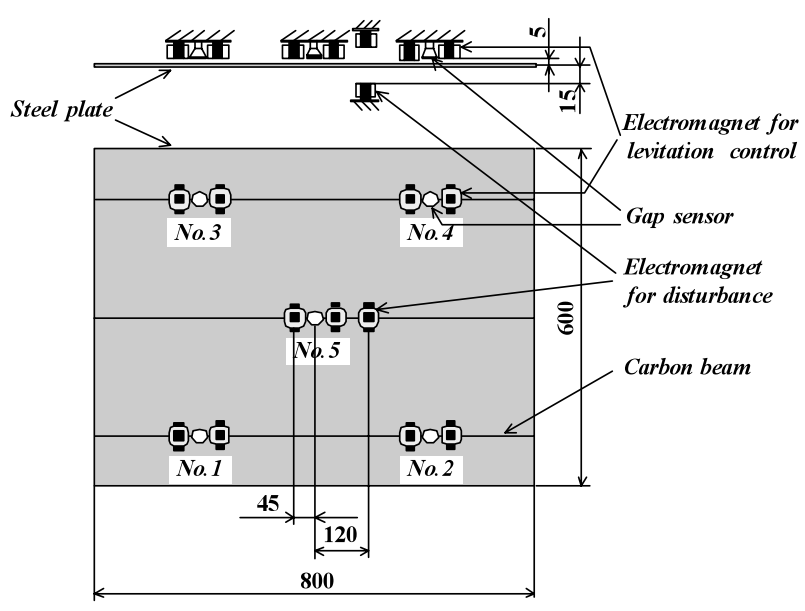

Fig. 2 Experimental apparatus

eration of disturbance are placed at the antinode position of the first elastic mode vibration, so that the steel plate is held between these magnets on its top and bottom sides. By making random voltage signals using a function generator and then adding these signals to the electromagnet, an external force is generated from these electromagnets. Furthermore, the random noise output from the function generator is also used as the noise within the control signal to the electromagnet No.5.

\section{Modeling of Steel Plate}

\section{1 Distributed model}

In a distributed model, we adopt independent control; information on values of displacement, velocity and coil current of the electromagnet detected at one position are fed back only to the same electromagnet. Accordingly, as shown in Fig. 3, the steel plate is divided into 5 hypothetical masses and each part is modeled as a lumped constant system.

When a steel plate is supported by the static electromagnetic attractive force of an electromagnet, an equilibrium state is gained where the plate is levitated at a constant height. Designating the displacement of the steel plate in the vertical direction of this state as $z_{n}$ ( $n$ corresponds to the numbers $1-5$ in Fig. 1), the equation of motion is given as follows.

$$
m_{z} \ddot{z}_{n}=2 f_{l n}
$$

Where $m_{z}=1 / 5 m, m$ : mass of the steel plate, $m_{z}$ : mass of the steel plate into five $[\mathrm{kg}]$, and $f_{l n}$ : dynamic magnetic force $[\mathrm{N}]$.

If deviation around the static equilibrium state is very small, the characteristic equations of the electromagnet are linearized as

$$
\begin{aligned}
& f_{l n}=\frac{F_{n}}{Z_{n}} z_{n}+\frac{F_{n}}{I_{n}} i_{n} \\
& \dot{i}_{n}=-\frac{L_{\text {eff }} I_{n}}{L_{z} Z_{n}^{2}} \dot{z}_{n}-\frac{R_{z}}{2 L_{z}} i_{n}+\frac{1}{2 L_{z}} v_{n}
\end{aligned}
$$

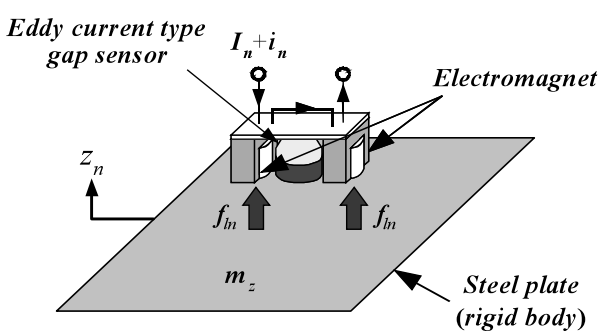

Fig. 3 Theoretical model of levitation control of a steel plate

$$
L_{z}=\frac{L_{e f f}}{Z_{n}}+L_{l e a}
$$

where $F_{n}$ : magnetic force of the coupled magnets in the equilibrium state $[\mathrm{N}], Z_{n}$ : gap between the steel plate and electromagnet in the equilibrium state $[\mathrm{m}], I_{n}$ : current of the coupled magnets in the equilibrium state $[\mathrm{A}], i_{n}$ : dynamic current of the coupled magnets $[\mathrm{A}], L_{z}$ : inductance of one magnet coil in the equilibrium state $[\mathrm{H}], R_{z}$ : resistance of the coupled magnet coils $[\Omega], v_{n}$ : dynamic voltage of the coupled magnets [V], $L_{e f f}$ : the component of effective inductance of one magnet coil in inverse proportion to the distance between the electromagnet and the plate $[\mathrm{Hm}]$, and $L_{\text {lea }}$ : leakage inductance of the one magnet coil [H].

\subsection{Continuous model ${ }^{(4)}$}

In a continuous model, integrated control is carried out by calculating 15 values, i.e., (1) Values of displacement, (2) Velocity, (3) Electromagnetic coil currents, all of which detected at each position of the five electromagnets. In this model, the motion of the steel plate is calculated from the equations of its elastic vibration. Supporting the plate by the static attracting force of each magnet creates an equilibrium levitation state, where the steel plate maintains a certain distance from the electromagnets. The equation of small vertical motion around the equilibrium state of the steel plate subjected to magnetic forces is expressed as follows.

$$
\begin{aligned}
& \rho h \frac{\partial^{2}}{\partial t^{2}} z+\frac{C h^{3}}{12} \frac{\partial}{\partial t} \nabla^{4} z+D \nabla^{4} z \\
& \quad=\sum_{n=1}^{5} f_{c n}(t)\left\{\delta\left(x-x_{a 1 n}\right) \delta\left(y-y_{a 1 n}\right)\right. \\
& \left.\quad+\delta\left(x-x_{a 2 n}\right) \delta\left(y-y_{a 2 n}\right)\right\}+w(t) \delta\left(x-x_{d 1 n}\right) \delta\left(y-y_{d 1 n}\right)
\end{aligned}
$$

$$
\nabla^{4}=\frac{\partial^{4}}{\partial x^{4}}+2 \frac{\partial^{4}}{\partial x^{2} \partial y^{2}}+\frac{\partial^{4}}{\partial y^{4}}
$$

Where $\rho$ : density $\left[\mathrm{kg} / \mathrm{m}^{3}\right], h$ : thickness $[\mathrm{m}], C$ : internal damping coefficient $\left[\mathrm{Ns} / \mathrm{m}^{2}\right], D=E h^{3} / 12\left(1-v^{2}\right)[\mathrm{Nm}]$, $v$ : Poisson ratio, $E$ : Young's modulus $\left[\mathrm{N} / \mathrm{m}^{2}\right], f_{c n}(t)$ : dynamic magnetic force at the $n$-th coupled magnets $[\mathrm{N}], t$ : time $[\mathrm{s}], z(x, y)$ : vertical displacement $[\mathrm{m}], x, y, z$ : coordinate axes indicated in Fig. $1[\mathrm{~m}], x_{a 1 n}, x_{a 2 n}, y_{a 1 n}, y_{a 2 n}$ : location of the $n$-th coupled magnets $(n=1-5)[\mathrm{m}], \delta()$ : Dirac delta function $[1 / \mathrm{m}], w(t)$ : dynamic magnetic force 
at the disturbance $[\mathrm{N}], x_{d 1 n}, y_{d 1 n}$ : position of the electromagnets for disturbance $[\mathrm{m}]$.

The characteristic equations of the electromagnets can be derived in the same way as distributed model, i.e.,

$$
\begin{aligned}
& f_{c n}=\frac{F_{n}}{Z_{n}} z\left(x_{s n}, y_{s n}\right)+\frac{F_{n}}{I_{n}} i_{n} \\
& \dot{i}_{n}=-\frac{L_{e f f} I_{n}}{L_{z} Z_{n}^{2}} \dot{z}\left(x_{s n}, y_{s n}\right)-\frac{R_{z}}{2 L_{z}} i_{n}+\frac{1}{2 L_{z}} v_{n}
\end{aligned}
$$

where $x_{s n}, y_{s n}$ : position of the $n$-th sensor [m]. However it must be taken into consideration that noise is added to the control voltage. Therefore equation of electromagnetic circuit current for the 5th electromagnet is given as follows.

$$
\dot{i}_{5}=-\frac{L_{e f f} I_{5}}{L_{z} Z_{0}^{2}} \frac{d}{d t} z\left(x_{s 5}, y_{s 5}\right)-\frac{R_{z}}{2 L_{z}} i_{5}+\frac{1}{2 L_{z}}\left(v_{5}+v_{w 5}\right)
$$

where $v_{w 5}$ : noise added to the electromagnetic coil [V].

\section{State Equation}

\section{1 State equation for distributed model}

Using the state vector, the Eqs. (1) - (4) are written as the following state equations:

$$
\begin{aligned}
\dot{z}_{l n} & =\boldsymbol{A}_{\text {ln }} z_{\text {ln }}+\boldsymbol{B}_{\text {ln }} v_{l n} \\
z_{\text {ln }} & =\left[\begin{array}{ccc}
z_{l n} & \dot{z}_{l n} & i_{l n}
\end{array}\right]^{\mathrm{T}} \\
\boldsymbol{A}_{\text {ln }} & =\left[\begin{array}{ccc}
0 & 1 & 0 \\
\frac{2 F_{n}}{m_{z} Z_{n}} & 0 & \frac{2 F_{n}}{m_{z} I_{n}} \\
0 & -\frac{L_{\text {eff }}}{L_{z}} \cdot \frac{I_{n}}{Z_{n}^{2}} & -\frac{R_{z}}{2 L_{z}}
\end{array}\right] \\
\boldsymbol{B}_{\text {ln }} & =\left[\begin{array}{lll}
0 & 0 & \frac{1}{2 L_{z}}
\end{array}\right]^{\mathrm{T}}
\end{aligned}
$$

\subsection{State equation for continuous model}

The vertical displacement of a plate can be expanded to an infinite series of a space-dependent eigenfunction $\phi_{i}(x, y)$ multiplied by the time-dependent normal coordinate. The eigenfunctions of the plate are assumed to be products of the elastic beam eigenfunctions of the $x$ and $y$-coordinates. The function of $y$-coordinate $Y_{n n}(x)$ $(n n=1,2, \ldots)$ satisfies the free-free boundary condition, and the function of the $x$-coordinate is expressed in rigid modes (parallel and rotational motions) $X_{1}(y), X_{2}(y)$ only. In addition, since the number of sensors used in this experiment is 5 , we selected $M=5$ for the control in which consideration is given to the 5 th mode (1st elastic mode). The mode-expansion equations are given as follows.

$$
\begin{aligned}
& z(x, y)=\sum_{i=1}^{M} \phi_{i}(x, y) \xi_{i}(t) \\
& \phi_{i}(x, y)=X_{m m}(x) \cdot Y_{n n}(y) \quad(m m, n n=1,2, \cdots) \\
& X_{1}(x)=1, \quad X_{2}(y)=\frac{\sqrt{3}}{a}(2 x-a) \\
& Y_{1}(y)=1, \quad Y_{2}(y)=\frac{\sqrt{3}}{b}(2 y-b)
\end{aligned}
$$

$$
\begin{aligned}
& Y_{n n}(y)=\cos \frac{\lambda_{y n n}}{b} y+\cosh \frac{\lambda_{y n n}}{b} y \\
& +\frac{\sin \lambda_{y n n}+\sinh \lambda_{y n n}}{\cos \lambda_{y n n}-\cosh { }_{y n n}}\left(\sin \frac{\lambda_{y n n}}{b} y+\sinh \frac{\lambda_{y n n}}{b} y\right) \\
& \cosh \lambda_{y n n} \cdot \cos \lambda_{y n n}=1 \quad(n n=3,4, \cdots) \\
& f_{y n n}=\frac{1}{2 \pi}\left(\frac{\lambda_{y n n}}{b}\right)^{2} \sqrt{\frac{D}{\rho h}}
\end{aligned}
$$

State variables of the system are normal coordinates of vertical displacement of the plate $\xi_{M}$, The control input of the system is the dynamic voltages of the magnets $\boldsymbol{v}_{\boldsymbol{c}}$. Output variables of the system are vertical displacements $z_{c}$. Using the state, control and output vectors, the forgoing Eqs. (5) - (8) can be written in the following state and output equations:

$$
\begin{aligned}
& \dot{\boldsymbol{\xi}}_{M}=\boldsymbol{A}_{\xi M} \boldsymbol{\xi}_{M}+\boldsymbol{B}_{\xi M}\left(\boldsymbol{v}_{c}+\boldsymbol{v}_{w}\right)+\boldsymbol{G}_{\xi M} w \\
& z_{c}=C_{\xi M} \boldsymbol{\xi}_{M} \\
& \boldsymbol{\xi}_{M}=\left[\begin{array}{lll}
\xi_{1} \cdots \xi_{M} & \dot{\xi}_{1} \cdots \dot{\xi}_{M} & i_{1} \cdots i_{5}
\end{array}\right]^{\mathrm{T}} \\
& \boldsymbol{v}_{\boldsymbol{c}}=\left[v_{1} \cdots v_{5}\right]^{\mathrm{T}} \\
& \boldsymbol{v}_{w}=\left[\begin{array}{lllll}
0 & 0 & 0 & 0 & v_{w 5}
\end{array}\right]^{\mathrm{T}} \\
& z_{c}=\left[\begin{array}{ccc}
z_{1} \cdots z_{5} & \dot{z}_{1} \cdots \dot{z}_{5} & i_{1} \cdots i_{5}
\end{array}\right]^{\mathrm{T}} \\
& \boldsymbol{A}_{\xi M}=\left[\begin{array}{ccc}
\mathbf{0} & \boldsymbol{I} & \mathbf{0} \\
\boldsymbol{A}_{21} & \boldsymbol{A}_{22} & \boldsymbol{A}_{23} \\
\mathbf{0} & \boldsymbol{A}_{32} & \boldsymbol{A}_{33}
\end{array}\right], \quad \boldsymbol{B}_{\xi M}=\left[\begin{array}{c}
\mathbf{0} \\
\mathbf{0} \\
\boldsymbol{B}_{31}
\end{array}\right], \\
& \boldsymbol{G}_{\xi M}=\left[\begin{array}{c}
\mathbf{0} \\
\boldsymbol{G}_{21} \\
\mathbf{0}
\end{array}\right], \quad \boldsymbol{C}_{\xi M}=\left[\begin{array}{ccc}
\boldsymbol{C}_{11} & \mathbf{0} & \mathbf{0} \\
\mathbf{0} & \boldsymbol{C}_{22} & \mathbf{0} \\
\mathbf{0} & \mathbf{0} & \boldsymbol{I}
\end{array}\right]
\end{aligned}
$$

here, details of Eqs. (11) and (12) are omitted due to space limitations ${ }^{(4)}$. Figure 4 shows the shape of the steel plate from 1 st to 5 th modes considered in the control system. In this figure, $\phi_{1}$ shows translational motion mode, $\phi_{2}$ rolling mode, $\phi_{3}$ pitching mode, $\phi_{4}$ twisting mode approximately set multiplying two rigid body modes; 2 nd and 3rd modes, and $\phi_{5}$ shows 1 st elastic mode.
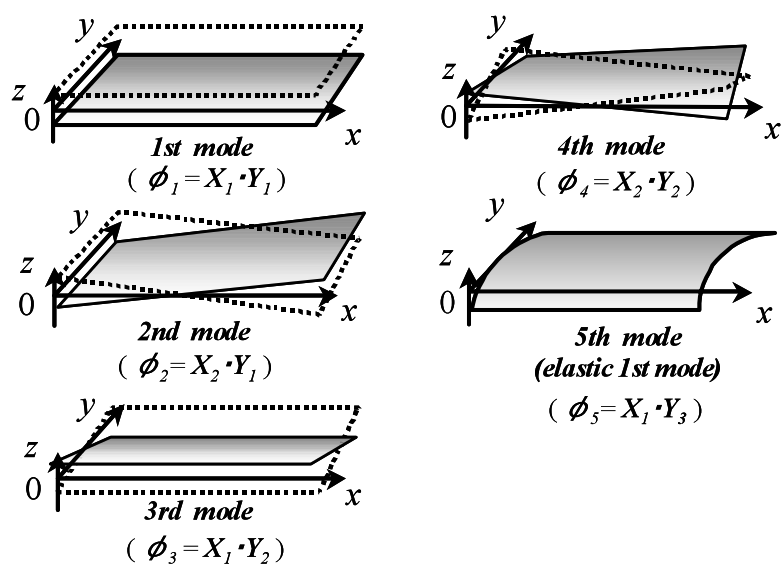

Fig. 4 Mode shape of the magnetically levitated steel plate for the continuous model 


\section{Control Theory}

\subsection{Optimal control in the discrete time system ${ }^{(9)}$}

In this study, a control system is constructed using a discrete time system; therefore, the evaluation function of a continuous system is digitized, and the optimal control law is obtained based on the optimal control theory of the discrete time system. The following discrete time system is hereby considered.

$$
\begin{aligned}
& \boldsymbol{z}_{d}(i+1)=\boldsymbol{\Phi} \boldsymbol{z}_{d}(i)+\boldsymbol{\Gamma} \boldsymbol{v}_{d}(i) \\
& \boldsymbol{\Phi}=\exp \left(\boldsymbol{A} T_{s}\right), \quad \boldsymbol{\Gamma}=\left\{\int_{0}^{T_{s}}[\exp (\boldsymbol{A} \tau)] d \tau\right\} \boldsymbol{B}
\end{aligned}
$$

In the case of a distributed model: $\boldsymbol{A}=\boldsymbol{A}_{l n}, \boldsymbol{B}=\boldsymbol{B}_{l n}$. In the case of a continuous model: $\boldsymbol{A}=\boldsymbol{A}_{\xi M}, \boldsymbol{B}=\boldsymbol{B}_{\xi M}$. Here, the evaluation function of the discrete time system is expressed as follows.

$$
\begin{aligned}
& \boldsymbol{J}_{d}=\sum_{i=0}^{\infty}\left[\boldsymbol{z}_{d}(i)^{\mathrm{T}} \boldsymbol{Q}_{d} \boldsymbol{z}_{d}(i)+\boldsymbol{v}_{d}(i)^{\mathrm{T}} \boldsymbol{r}_{d} \boldsymbol{v}_{d}(i)\right] \\
& \boldsymbol{M}=\boldsymbol{\Phi}^{\mathrm{T}} \boldsymbol{M} \boldsymbol{\Phi}+\boldsymbol{Q}_{d}-\boldsymbol{\Phi}^{\mathrm{T}} \boldsymbol{M} \boldsymbol{\Gamma}\left(\boldsymbol{r}_{d}+\boldsymbol{\Gamma}^{\mathrm{T}} \boldsymbol{M} \boldsymbol{\Gamma}\right)^{-1} \boldsymbol{\Gamma}^{\mathrm{T}} \boldsymbol{M \Phi}
\end{aligned}
$$

$$
\begin{aligned}
& \boldsymbol{v}_{d}^{o}=-\boldsymbol{F}_{d} \boldsymbol{z}_{d} \\
& \boldsymbol{F}_{d}=\left(\boldsymbol{r}_{d}+\boldsymbol{\Gamma}^{\mathrm{T}} \boldsymbol{M} \boldsymbol{\Gamma}\right)^{-1} \boldsymbol{\Gamma}^{\mathrm{T}} \boldsymbol{M \Phi}
\end{aligned}
$$

Where $\boldsymbol{Q}_{d}$ and $\boldsymbol{r}_{d}$ are weighting coefficients, $\boldsymbol{M}$ is the solution of the algebraic matrix Riccati equation, and $T_{s}$ is a sampling interval. MATLAB command "lqrd" was used to solve Eq. (15) and the digital controller was designed by using SIMLINK in the DSP.

\subsection{Sliding mode control in the discrete time sys- tem $^{(8)}$}

Similar to the previous section 5.1, the discrete time system of Eq. (13) is considered here. Now we designate the switching hyperplane as $\boldsymbol{S}_{d}$, and express the switching function of input as

$$
\boldsymbol{\sigma}(i)=\boldsymbol{S}_{d} z_{d}(i)
$$

The equivalent control input is given as

$$
\boldsymbol{v}_{\boldsymbol{e q}}^{s}(i)=-\left(\boldsymbol{S}_{d} \boldsymbol{\Gamma}\right)^{-1} \boldsymbol{S}_{d}(\boldsymbol{\Phi}-\boldsymbol{I}) \boldsymbol{z}_{d}(i)
$$

Substituting Eq. (19) into Eq. (13), the equivalent control system can be expressed as

$$
\boldsymbol{z}_{d}(i+1)=\left\{\boldsymbol{\Phi}-\boldsymbol{\Gamma}\left(\boldsymbol{S}_{d} \boldsymbol{\Gamma}\right)^{-1} \boldsymbol{S}_{d}(\boldsymbol{\Phi}-\boldsymbol{I})\right\} \boldsymbol{z}_{d}(i)
$$

Here, $\boldsymbol{S}_{d}$ should be selected such that the system represented by Eq. (20) becomes stable. In this study, we use a method of utilizing the zero point of the system, and the optimal control theory of the discrete time system is applied in order to obtain $S_{d}$.

$$
\boldsymbol{S}_{d}=\left(\boldsymbol{r}_{d}^{s}+\boldsymbol{\Gamma}^{\mathrm{T}} \boldsymbol{M}^{s} \boldsymbol{\Gamma}\right)^{-1} \boldsymbol{\Gamma}^{\mathrm{T}} \boldsymbol{M}^{s} \boldsymbol{\Phi}_{\varepsilon}
$$

Where $\boldsymbol{r}_{d}^{s}$ is weighting coefficients for control input. $\boldsymbol{M}^{s}$ is a solution of Eq. (13).

Next, a control input which converges the state into a hyperplane and generates the sliding mode is considered.
In this study, we design a sliding mode control for the discrete time system, wherein chattering is suppressed. The sliding mode control law used to satisfy this condition is described below.

$$
\left.\begin{array}{l}
\boldsymbol{v}_{d}^{s}(i)=\boldsymbol{v}_{e q}^{s}(i)+\boldsymbol{v}_{\boldsymbol{n} \boldsymbol{l}}^{s}(i) \\
\boldsymbol{v}_{\boldsymbol{e q}}^{s}(i)=-\left(\boldsymbol{S}_{d} \boldsymbol{\Gamma}\right)^{-1} \boldsymbol{S}_{d}(\boldsymbol{\Phi}-\boldsymbol{I}) \boldsymbol{z}_{d}(i) \\
\boldsymbol{v}_{\boldsymbol{n} \boldsymbol{l}}^{s}(i)=-\{\alpha(i)+\beta(i)\} \operatorname{sgn}\{\boldsymbol{\sigma}(i)\}
\end{array}\right\}
$$

$F_{\text {max }}$ : Maximum value of disturbance.

Explained below are the factors that satisfy the matching condition. $\boldsymbol{h}$ is defined as a function that includes systematic uncertainties and/or disturbances. When disturbance $\boldsymbol{h}$ exists in the range space of $\boldsymbol{B}_{\xi M}$ in the Eq. (11),

$$
\boldsymbol{h} \subset \operatorname{Range}\left(\boldsymbol{B}_{\xi M}\right)
$$

i.e.

$$
\boldsymbol{h}=\boldsymbol{B}_{\xi M} \lambda
$$

Which means $\boldsymbol{h}$ satisfies the matching condition ${ }^{(7)}$. This shows that uncertainties and/or disturbances in all models come in through the same channel as the control input. Which is: under the system in this thesis, all disturbances within the control signals, such as the resistance due to heat generation in the electromagnet, and power source noise, are to satisfy the matching condition. In addition, $v_{w 5}$ in the Eq. (11) can be expressed as follows, thus proving to satisfy the matching condition.

$$
\begin{aligned}
& \boldsymbol{B}_{\xi M} \boldsymbol{v}_{w} \subset \operatorname{Range}\left(\boldsymbol{B}_{\xi M} \boldsymbol{v}_{c}\right) \\
& {\left[\begin{array}{c}
\mathbf{0} \\
\mathbf{0} \\
\boldsymbol{B}_{31}
\end{array}\right] \boldsymbol{v}_{w} \subset \text { Range }\left(\left[\begin{array}{c}
\mathbf{0} \\
\mathbf{0} \\
\boldsymbol{B}_{31}
\end{array}\right] \boldsymbol{v}_{c}\right)}
\end{aligned}
$$

However the Eq. (26) cannot be realized when disturbance $\boldsymbol{h}$ does not exist in the range space of $\boldsymbol{B}_{\xi M}$. Under the system in this thesis, a disturbance such as external forces is the primal case that does not satisfy the condition. I.e. $w$ in the Eq. (11) can be given as follows, not satisfying the matching condition.

$$
\begin{aligned}
& \boldsymbol{G}_{\xi M} w \not \subset \text { Range }\left(\boldsymbol{B}_{\xi M} \boldsymbol{v}_{\boldsymbol{c}}\right) \\
& {\left[\begin{array}{c}
\mathbf{0} \\
\boldsymbol{G}_{21} \\
\mathbf{0}
\end{array}\right] w \not \subset \text { Range }\left(\left[\begin{array}{c}
\mathbf{0} \\
\mathbf{0} \\
\boldsymbol{B}_{31}
\end{array}\right] \boldsymbol{v}_{c}\right)}
\end{aligned}
$$

\section{Control Experiment and Numerical Simulation}

\subsection{Specifications of the experimental apparatus}

The specifications of the system are shown in Table 1 .

\subsection{Setting the comparative standard for control} systems

In this study, we examine the vibration-suppression performance during levitation of a flexible steel plate for a total of 4 cases: optimal control applied to the distributed 
Table 1 Symbols and values

\begin{tabular}{|c|c|c|c|}
\hline Paragraph & Symbol & Paragraph & Symbol \\
\hline \multirow{10}{*}{3.1} & $m=1.08 \mathrm{~kg}$ & \multirow{10}{*}{3.2} & $\rho=7500 \mathrm{~kg} / \mathrm{m}^{3}$ \\
\hline & $Z_{n}=5 \mathrm{~mm}$ & & $E=217 \mathrm{GPa}$ \\
\hline & $F_{1} \sim F_{4}=1.85 \mathrm{~N}$ & & $v=0.3$ \\
\hline & $F_{5}=3.21 \mathrm{~N}$ & & $C=2.49 \times 10^{8} \mathrm{Ns} / \mathrm{m}$ \\
\hline & $I_{1} \sim I_{4}=0.51 \mathrm{~A}$ & & $x_{s 1}=x_{s 3}=185 \mathrm{~mm}$ \\
\hline & $I_{5}=0.67 \mathrm{~A}$ & & $x_{s 2}=x_{s 4}=615 \mathrm{~mm}$ \\
\hline & $R_{z}=20.6 \Omega$ & & $x_{s 5}=400 \mathrm{~mm}$ \\
\hline & $L_{e f f}=1.8 \times 10^{-4} \mathrm{Hm}$ & & $y_{s l}=y_{s 3}=83 \mathrm{~mm}$ \\
\hline & $L_{\text {lea }}=0.1891 \mathrm{H}$ & & $y_{s 2}=y_{s 4}=517 \mathrm{~mm}$ \\
\hline & $L_{z}=0.225 \mathrm{H}$ & & $y_{s 5}=300 \mathrm{~mm}$ \\
\hline 5.1 & $T_{s}=1 \mathrm{~ms}$ & & \\
\hline
\end{tabular}

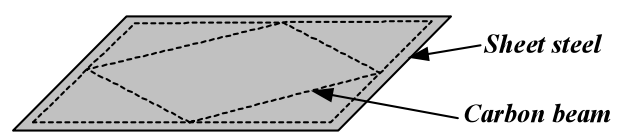

Fig. 5 Rigid steel plate
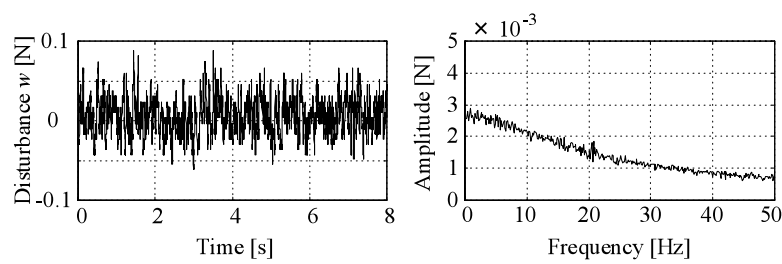

Fig. 6 Time history and amplitude spectrum of disturbance from electromagnet

model and to the continuous model, and sliding mode control applied to each of the two models. To compare the performance of each control system, a certain standard must be determined. Hence we are to assume a situation where neither modeling nor the control theory bear any differences on the systems, and each system is designed to maintain identical levitation performance, after which we are to examine how the control performance changes according to modeling or control theory under this criterion.

Carbon fiber-made pipes are attached as shown in Fig. 5 and a criterion is set so that the levitation performance remains the same when an external-force disturbance is input to the steel plate, which is regarded as a rigid-body with no elastic vibrations. As this kind of disturbance does not satisfy the matching condition, the superiority peculiar to sliding mode control does not theoretically appear. Therefore it can be considered the most adequate disturbance in order to set the criterion in comparing the performance of each control system. It is also adequate as a criterion to compare the control efficiency that varies in accordance to modeling changes, as carbon fiber-made pipes canceling the elastic vibrations theoretically invalidates the superiority of the continuous model over such type of vibration.

An external force $w$ (calculated from the coil current of the electromagnet), as shown in Fig. 6, is applied to the rigid plate levitated using each control method. This external force is generated by adding random voltage sig-
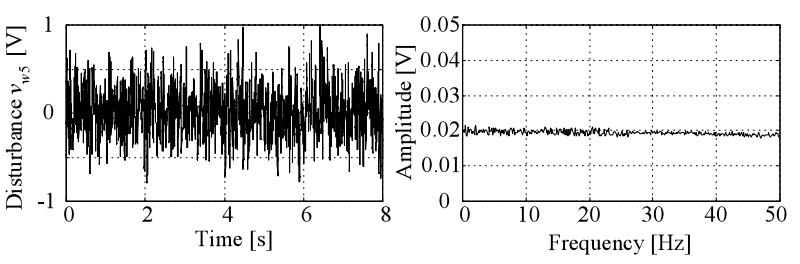

Fig. 7 Time history and amplitude spectrum of disturbance into the control input

nals to the electromagnet, the signals having such a characteristic that maintains the frequency between $0-50 \mathrm{~Hz}$. Upon expecting vibration modes such as rolling and pitching modes under this condition, there ought to be difficulties comparing the performance of each control system. Therefore electromagnet for disturbance is installed nearby the magnet set at the center of the steel plate, which is also a position where the vibration mode in the $y$-axis direction rarely occurs.

Under these circumstances stated above, the parameters of each control system are set such that the standard deviation of the displacement at the center of the steel plate is within an error range of $\pm 5 \%$ for $1.0 \times 10^{-5} \mathrm{~m}$ during the application of any control method.

The weighting matrices of optimal control (Eq. (14)) are

- $\quad$ Distributed model: $\boldsymbol{Q}_{d}^{l, o p t}=\boldsymbol{I}, \boldsymbol{r}_{d}^{l, o p t}=1$.

- Continuous model: $\boldsymbol{Q}_{d}^{c, o p t}=\boldsymbol{I}, \boldsymbol{r}_{d}^{c, o p t}=\boldsymbol{I}$.

The weighting matrices (Eq. (14)) used for determining the switching function $\boldsymbol{S}_{d}$ in sliding mode control, and parameters in the nonlinear input terms in Eq. (23) are

- $\quad$ Distributed model: $\boldsymbol{Q}_{d}^{l, s m c}=\boldsymbol{I}, \boldsymbol{r}_{d}^{l, s m c}=1, \eta=0.05$, $\beta=3$.

- Continuous model: $\boldsymbol{Q}_{d}^{c, s m c}=\boldsymbol{I}, \boldsymbol{r}_{d}^{c, s m c}=\boldsymbol{I}, \eta=0.05$, $\beta=3$.

In the following sections, we discuss the cases in which the flexible steel plate described in chapter 2 (Figs. 1 and 2) is levitated using control systems designed with the above-described parameters.

\subsection{Setting of disturbance input}

Below is the study examining each control performance when there are inputs of a disturbance that does/ does not satisfy the matching condition. External force $w$ shown in Fig. 6 is to be used as a disturbance that does not satisfy the matching condition.

As a disturbance that does satisfy the matching condition, noise within the control electric currents is adopted, this noise being forcibly added to the control signal of electromagnet No.5 installed at the center of the steel plate. Shown in Fig. 7 are an example of time response (an actual measurement) of the voltage $v_{w 5}$ used as a disturbance in this case and its amplitude spectrum. This voltage has a characteristic that maintains the frequency between $0-50 \mathrm{~Hz}$, alike the random voltage signal used to generate the disturbance shown in Fig. 6. The height of volt- 
age $v_{w 5}$ is decided as follows; arrange the voltage signal coming from the function generator so that the standard deviations of the displacement at the center of the plate show approximate values (within an error of $10 \%$ ) when either external force $w$ in Fig. 6 or disturbance voltage $v_{w 5}$ is input into the steel plate levitated with the application of distributed model + optimal control.

\section{4 How to conduct numerical simulation}

Numerical simulation is conducted by adopting the parameter shown in Table 1 and the control parameter decided in section 6.2, and substituting control voltage $\boldsymbol{v}_{d}^{o}$ (Eq. (16)) or $\boldsymbol{v}_{d}^{s}$ (Eq. (22)) for $\boldsymbol{v}_{c}$ in Eq. (11). $\boldsymbol{v}_{d}^{o}$ and $\boldsymbol{v}_{d}^{s}$ are calculated from each control theory in chapter 5 . Vibration modes from 1st to 14th (Eq. (10), $M=14$ ) are taken into consideration. Also, to imitate an external force from the disturbance electromagnet, time response shown in Fig. 6 is input to $w$ in Eq. (11) as a numerical datum. As to the disturbance added to the control signal, time response of the disturbance as voltage, shown in Fig. 7, is input to $v_{w 5}$ in Eq. (11).

\subsection{Vibration-suppression performance when dis- turbance is within electromagnetic circuit cur- rent}

When an electromagnet levitations a steel plate, the change in the resistance due to heat generated in the electromagnet, as well as power source noise, significantly affects the control performance. When an uncertainty is included in the control system, it is practically very important to design a control system that accommodates such uncertainties. Therefore, we here investigate the effect of the suppression of uncertainties included in the same channel as control signals, namely, a disturbance which satisfies the matching condition of sliding mode control.

In order to intimate uncertainties within the electromagnetic circuit current, the disturbance shown in Fig. 7 is forcibly added to the control signal No.5. We have confirmed by both pre-experiment and numerical simulation that third or higher mode vibrations are rarely excited, which is guaranteed by the characteristics of the object to be controlled (steel plate). Therefore in this study, we mainly discuss the 1st elastic mode vibration, which most frequently occurs.

Figure 8 shows experimental results, and Fig. 9 shows the results of the numerical simulation under the same conditions. In Figs. 8 and 9, examples of the results of the time history and amplitude spectrum of the displacement at the center of the steel plates are shown: chart (a) and (b) represent the cases in which optimal control and sliding mode control are respectively applied to the distributed model, chart (c) and (d) represent the cases in which optimal control and sliding mode control are respectively applied to the continuous model. The zero on the vertical axis of the time history represents the position of equilibrium levitation. The experimental results basically agree
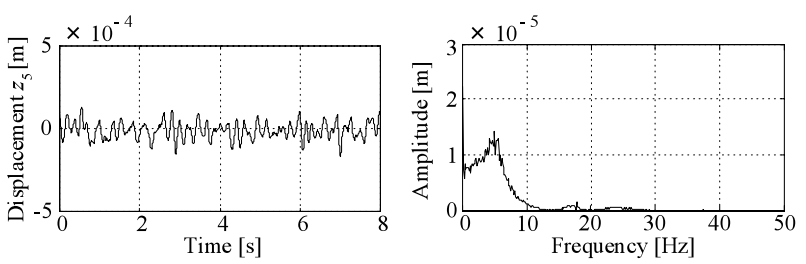

(a) Optimal control using distributed model
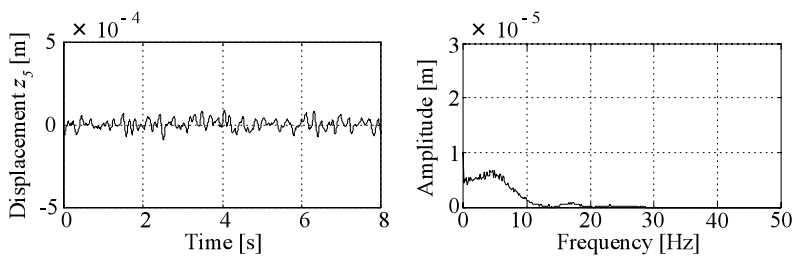

(b) Sliding mode control using distributed model
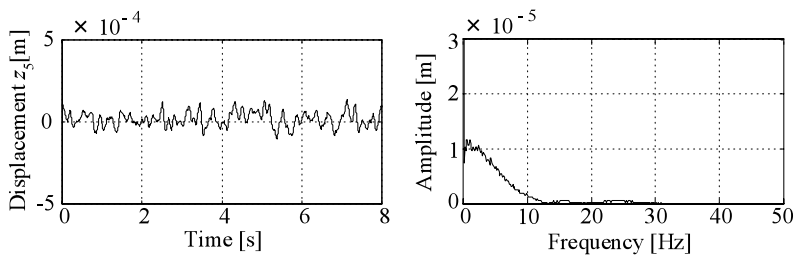

(c) Optimal control using continuous model
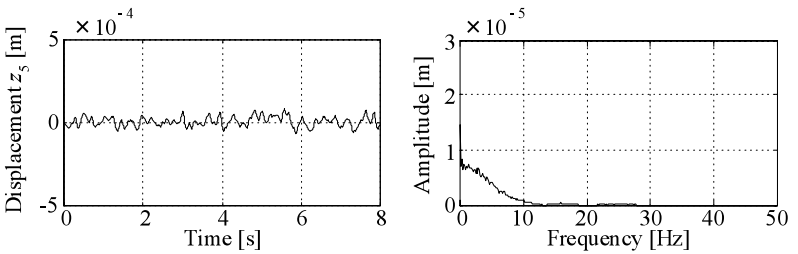

(d) Sliding mode control using continuous model

Fig. 8 Experimental results of time histories and amplitude spectrums of displacement at center of the steel plate under random excitation from disturbance into the control input

with the numerical simulation results, which indicates the validity of the models. Therefore, only experimental results are referred to in the following discussion.

First, we examine the differences between the control methods when the distributed model is used. When optimal control is applied (Fig. 8 (a)), elastic vibrations are excited on the steel plate due to the enforced disturbance applied to the control signal. A peak of the amplitude spectrum is observed at $4.75 \mathrm{~Hz}$, which is the frequency of the 1st elastic mode vibration of the steel plate used in this experiment. In contrast, when sliding mode control is applied (Fig. 8 (b)), the elastic vibration of the plate is suppressed. This is because the system is capable of canceling disturbances contained in the circuit current, which are difficult to remove with the conventional linear control theory. However, in sliding mode control, switching of the control input at an infinite speed is assumed; indicating that the theoretical control performance cannot be completely achieved even when the matching condition is satisfied. Consequently, vibration of the 1st elastic mode 

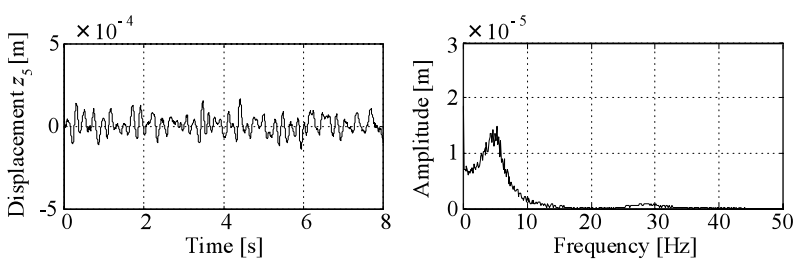

(a) Optimal control using distributed model
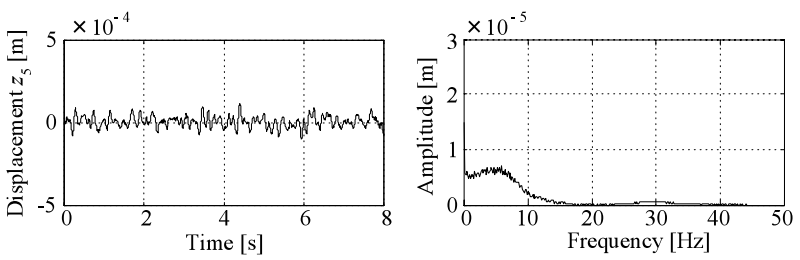

(b) Sliding mode control using distributed model
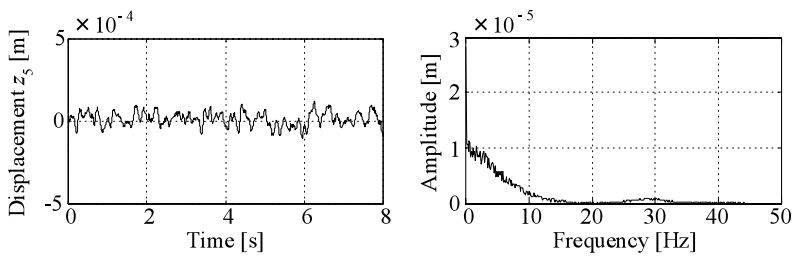

(c) Optimal control using continuous model
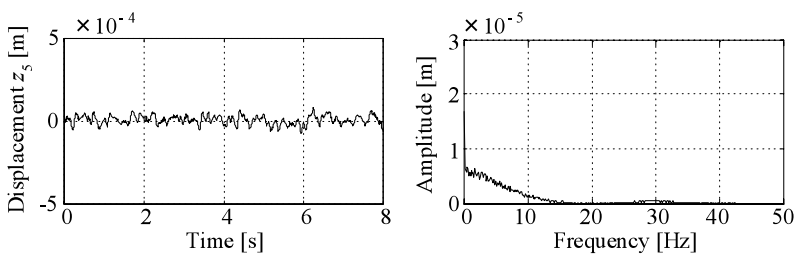

(d) Sliding mode control using continuous model

Fig. 9 Simulation results of time histories and amplitude spectrums of displacement at center of the steel plate under random excitation from disturbance into the control input

remains and a peak is observed in the spectrum, although slight.

Secondly, we examine differences in the modeling of thin steel plates. In Fig. 8 (c) and (d), due to the use of the continuous model that takes 1 st elastic mode vibration into consideration, no peak is observed in the amplitude spectrum, in comparison to the results in Fig. 8 (a) and (b). In particular, when sliding mode control is applied to the continuous model (Fig. 8(d)), the best control performance is obtained among the four cases, as cancellation of the disturbance contained in the control signals is canceled, and the suppression of the 1st elastic mode vibration can be achieved.

Table 2 summarizes the standard deviation of the time history shown in Fig. 8 (a) - (d). The standard deviation when optimal control is applied to the distributed model is set as the standard value (100\%) ((a) in Table 2), and the standard deviation in each case is expressed in percentage relative to this standard value. The standard deviations for the cases when sliding mode control is applied ((b) and (d) in Table 2) are reduced to around $60 \%$ of the
Table 2 Standard deviation and the relative ratio on the time histories in the case of the disturbance into the control input

\begin{tabular}{|l||c||c||c|}
\hline \multicolumn{1}{|l||}{ Modeling and control theory } & $\begin{array}{c}\text { Standard } \\
\text { deviation }\end{array}$ & Relative ratio \\
\hline \hline (a) & Distributed model + Optimal control & $5.15 \times 10^{-5} \mathrm{~m}$ & $100 \%$ \\
\hline (b) & Distributed model + Sliding mode control & $3.23 \times 10^{-5} \mathrm{~m}$ & $63 \%$ \\
\hline (c) & Continuous model + Optimal control & $4.25 \times 10^{-5} \mathrm{~m}$ & $83 \%$ \\
\hline (d) & Continuous model + Sliding mode control & $2.85 \times 10^{-5} \mathrm{~m}$ & $55 \%$ \\
\hline
\end{tabular}

standard value, indicating the superiority of sliding mode control, regardless of the steel-plate model used. In particular, when the distributed model is used, which is easy to be modeled, the vibration amplitude can be sufficiently suppressed by using sliding mode control ((b) in Table 2).

6.6 Performance of suppression of forced elastic vibration generated by external force

In the chapter above, we examined the uncertainties within the same channel as the control signal, or the control performance of the sliding mode control over disturbances that satisfies the matching condition. We here examine cases in which a steel plate during conveyance causes elastic vibrations, receiving unexpected external forces from sources other than the supporting electromagnets, or when there is an input of a disturbance does not satisfy the matching condition.

A disturbance as shown in Fig. 6 is added to the steel plate. A disturbance electromagnet is placed in the antinode position of the steel plate in the 1st elastic vibration mode as shown in Fig. 2, forcibly creating a situation where elastic vibration can be easily excited. Under such circumstances, if any vibration mode other than those shown in experiments in the last chapter is excited, it should be difficult to compare the result of the last chapter to the control performance with the input of a disturbance that does not satisfy the matching condition. Therefore the disturbance electromagnet is to be positioned as closely to the electromagnet at the center of the steel as possible, this being another reason for placing the disturbance electromagnet as is shown in Fig. 2.

When disturbance is imposed on a steel plate from sources other than the support electromagnet, the disturbance basically does not satisfy the matching condition of sliding mode control, as Eq. (28) shows. Nonami et al. ${ }^{(5)}$ showed that in case sufficient feedback of the current allows the first delay of the electromagnetic coil current to be neglected, the matching condition is fulfilled even when the disturbance is absent from the same channel as the control input. However, our study shows that the time constant of the electromagnets is $12.6 \mathrm{~ms}$, which is relatively large because it is equivalent to $6 \%$ of the period of the $1 \mathrm{st}$ mode vibration, $0.21 \mathrm{~s}$. Moreover, due to various restrictions of the system, feedback on the current cannot be given to the extent that the 1st delay can be ignored. 


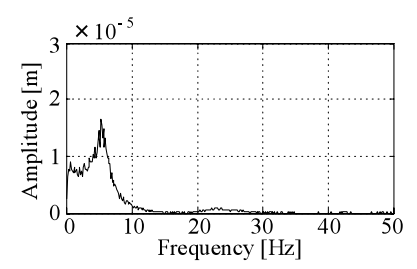

(a) Optimal control using distributed model

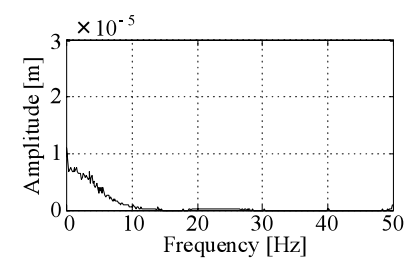

(c) Optimal control using continuous model

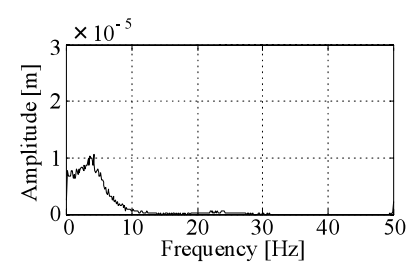

(b) Sliding mode control using distributed model

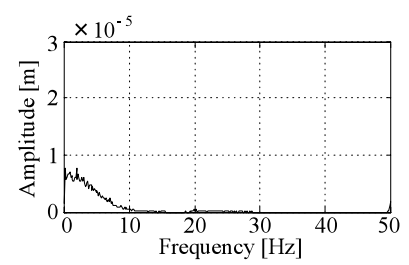

(d) Sliding mode control using continuous model

Fig. 10 Experimental results of amplitude spectrums of displacement at center of the steel plate under random excitation from the electromagnet for disturbance

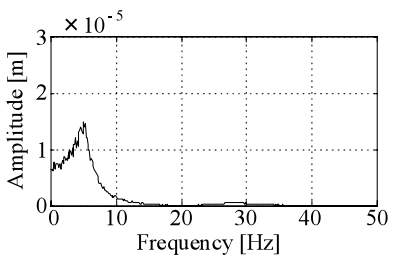

(a) Optimal control using distributed model

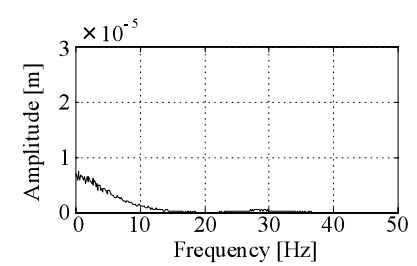

(c) Optimal control using continuous model

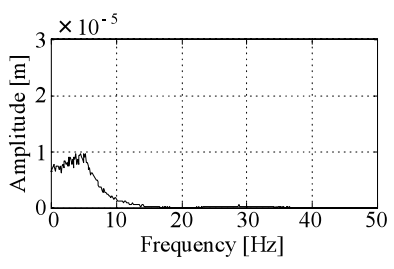

(b) Sliding mode control using distributed model

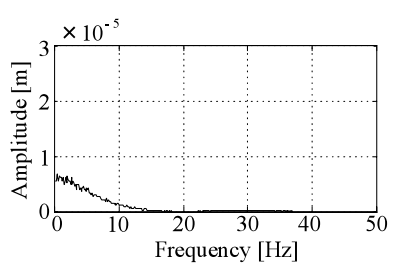

(d) Sliding mode control using continuous model

Fig. 11 Simulation results of amplitude spectrums of displacement at center of the steel plate under random excitation from the electromagnet for disturbance

As a result, it is considered that the complete matching condition does not hold true.

In Figs. 10 and 11, experimental and numerical simulation results for the two control methods are shown (only the amplitude spectrum of displacement); (a) -(d) represent the same cases as those in section 6.5, and the results show good agreement. In Fig. 10, when the distributed model is used (Fig. 10 (a) and (b)), a peak of the amplitude spectrum due to spillover of the 1st elastic mode vibration is observed with both control methods. Examining these results, it can be confirmed that a control system adopting sliding mode control (Fig. 10 (b)) does not have vibration control abilities unlike the system (Fig. 8(b)) in the last chapter, in case there is an input of a disturbance that

Table 3 Standard deviation and the relative ratio on the time histories in the case of the disturbance from the electromagnet

\begin{tabular}{|c||c||c||c|}
\hline \multicolumn{1}{|l||}{ Modeling and control theory } & $\begin{array}{c}\text { Standard } \\
\text { deviation }\end{array}$ & Relative ratio \\
\hline \hline (a) & Distributed model + Optimal control & $5.27 \times 10^{-5} \mathrm{~m}$ & $100 \%$ \\
\hline (b) & Distributed model + Sliding mode control & $4.48 \times 10^{-5} \mathrm{~m}$ & $85 \%$ \\
\hline (c) & Continuous model + Optimal control & $2.79 \times 10^{-5} \mathrm{~m}$ & $53 \%$ \\
\hline (d) & Continuous model + Sliding mode control & $2.69 \times 10^{-5} \mathrm{~m}$ & $51 \%$ \\
\hline
\end{tabular}

does not satisfy the matching condition. However, the system in Fig. 10 (b) shows some superiority, although slight, when comparing the two distributed models (Fig. 10 (a), (b)) both of which do not take elastic vibrations into consideration. This is due to the robustness against modeling errors that sliding mode control shows.

On the other hand, unlike Fig. 10 (a), (b), using the continuous models shown in Fig. 10 (c), (d) shows no significant differences. Under the circumstances where there is an input of a disturbance that does not satisfy the matching condition, this indicates that, when the vibration mode of the object to be controlled is taken into consideration in the controller, and therefore modeling errors do not occur, the sliding mode control does not show its superiority.

Table 3 summarizes the standard deviation of the time history of displacement, in the same way as to Table 2 in section 6.5. When the continuous model is used ((c) and (d) in Table 2), the deviation is reduced to approximately $50 \%$ of the standard ((a) in Table 3 ) in both control methods. When the continuous model is used ((c) and (d) in Table 3), the standard deviation is reduced to approximately $50 \%$ of the standard ((a) in Table 3) in both control methods. This result means that its vibration-suppression ability fully appears against 1 st elastic mode considered in the continuous model. From this, we can conclude that when an external force that does not satisfy the matching condition is applied, and therefore elastic vibration is forcibly excited, effective suppression of the elastic vibration can be ensured by the use of the continuous model.

6.7 Suppression performance of elastic vibration when two types of disturbance are simultaneously applied

We have examined two different cases, in section 6.5 discussing a case with the input of a disturbance that satisfies the matching condition, whereas in section 6.6 discussing one with the input of a disturbance that does not satisfy the matching condition. However in actual processes, such disturbances are expected to be applied simultaneously onto a steel plate. It cannot be assured how the control system would be influenced should disturbances under different conditions be simultaneously input. Therefore, in this section, we discuss cases where the two types of disturbances occur simultaneously.

Figure 12 shows experimental results when the dis- 


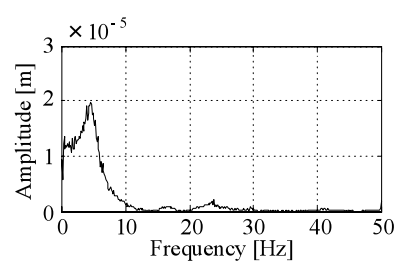

(a) Optimal control using distributed model

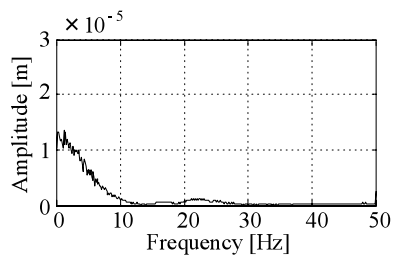

(c) Optimal control using continuous model

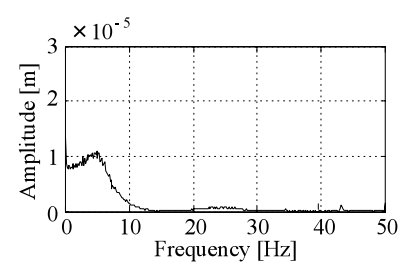

(b) Sliding mode control using distributed model

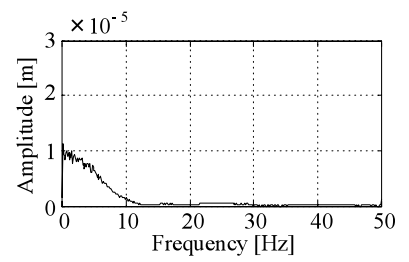

(d) Sliding mode control using continuous model

Fig. 12 Experimental results of amplitude spectrums of displacement at center of the steel plate under random excitation from disturbance into the control input and the electromagnet for disturbance

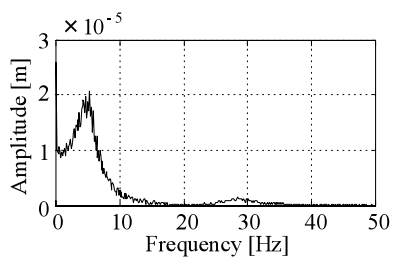

(a) Optimal control using distributed model

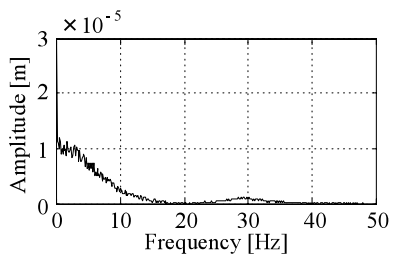

(c) Optimal control using continuous model

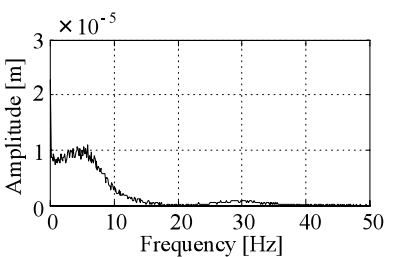

(b) Sliding mode control using distributed model

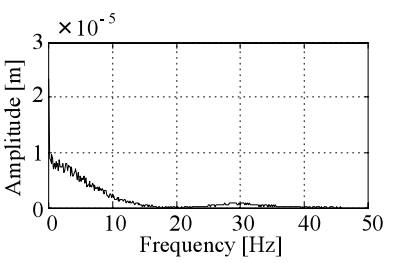

(d) Sliding mode control using continuous model

Fig. 13 Simulation results of amplitude spectrums of displacement at center of the steel plate under random excitation from disturbance into the control input and the electromagnet for disturbance

turbances shown in Figs. 6 and 7 are simultaneously input to the system. Figure 13 shows the numerical simulation results under the same conditions. The two sets of results are consistent.

Table 4 summarizes the standard deviation of the time history of displacement. Applying sliding mode control to the continuous model (d), suppression of the disturbance of the circuit signals as well as suppression of the 1st elastic mode vibration excited by external force is possible in a compounded manner. Therefore, superior suppression performance of the vibration has been realized, compared with other cases.

Table 4 Standard deviation and the relative ratio of the standard deviation on the time histories in the case of disturbance into the control input and the disturbance to the control input

\begin{tabular}{|l||c||c||c|}
\hline \multicolumn{1}{|l||}{ Modeling and control theory } & $\begin{array}{c}\text { Standard } \\
\text { deviation }\end{array}$ & Relative ratio \\
\hline \hline (a) & Distributed model + Optimal control & $7.28 \times 10^{-5} \mathrm{~m}$ & $100 \%$ \\
\hline (b) & Distributed model + Sliding mode control & $5.26 \times 10^{-5} \mathrm{~m}$ & $72 \%$ \\
\hline (c) & Continuous model + Optimal control & $4.83 \times 10^{-5} \mathrm{~m}$ & $66 \%$ \\
\hline (d) & Continuous model + Sliding mode control & $3.79 \times 10^{-5} \mathrm{~m}$ & $52 \%$ \\
\hline
\end{tabular}

On the other hand, the relative ratios in Table 4 consists with the respective values shown in Tables 2 and 3. For example, the average values of the relative ratio for case (b) in Table $2(63 \%)$ and Table $3(85 \%)$ are close to the relative ratio for case (b) in Table 4 (72\%). Namely, when the disturbances mentioned above are applied simultaneously, the characteristics of the electromagnetic levitation system of thin steel plates show linearity within the range of this experimental condition. Thus, we confirmed that it is possible to predict the result when such disturbances are applied simultaneously, on the basis of the results of the independent analyses of each disturbance, as described in sections 6.5 and 6.6.

\section{Conclusions}

Assuming actual processes wherein various disturbances are applied to an electromagnetically levitated thin steel plate, we evaluated the performance of a system along with modeling of the thin steel plate, by applying sliding mode control for vibration suppression. The following results were obtained.

( 1 ) By applying a discrete-time sliding mode control that suppresses chattering, it is possible to build a system that has outstanding vibration control performance, without exciting high-order elastic vibration mode.

(2) For an electromagnetic levitation system of thin steel plates, the application of sliding mode control to the distributed model, which can be fairly easily modeled, revealed the possibility of effective suppression of disturbances. In particular, sliding mode control was confirmed to be highly robust to uncertainties included in the circuit current of electromagnets. Since the use of a simple model can reduce the development cost of control systems, a result with industrial effectiveness has been obtained.

( 3 ) Our demonstration confirmed that ensuring superior control performance over disturbances that do not completely satisfy a matching condition is possible by the application of sliding mode control to the continuous model of a thin steel plate, although numerical manipulation is fairly complex. The results of this study are applicable to various factors that deteriorate the levitation stability of thin steel plates during actual conveyance processes, such as generation of heat in a long-hour use of the elec- 
tromagnets, various types of noise brought into the control signal, and elastic vibrations imposed on the plate, thereby enabling practically effective control systems.

(4) Modeling of thin steel plates has been considered difficult because they are flexible. However, in all the cases examined in this study, experimental results agreed well with numerical simulation results, confirming the adequacy of the numerical models used in this study. Furthermore, it has become possible to discuss situations that are difficult to examine experimentally, such as high-order elastic vibrations that are expected to occur due to the thinness of steel plates and various parameter changes in the object to be controlled. Thus, the present study has revealed developmental features useful for future research.

\section{References}

(1) Matsuda, K., Yoshihashi, M., Okada, Y. and Tan, A.C.C., Self-Sending Active Suppression of Vibration of Flexible Steel Sheet, Trans. ASME, Journal of Vibration and Acoustics, Vol.118 (1996), pp.469-473.

(2) Nakagawa, T., Hama, M. and Furukawa, T., Study of Magnetic Levitation Technique Applied to Steel Plate Production Line, IEEE Trans. Magn., Vol.36, No.5 (2000), pp.3686-3689.
( 3 ) Sase, M. and Torii, S., Magnetic Levitation Control with Real-Time Vibration Analysis Using Finite Element Method, International Journal of Applied Electromagnetics and Mechanics, Vol.13, Nos.14 (2001/2002), pp.129-136.

( 4 ) Oshinoya, Y. and Obata, T., Noncontact Vibration Control of a Magnetic Levitated Rectangular Thin Steel Plate, JSME Int. J., Ser.C, Vol.45, No.1 (2001), pp.6069.

( 5 ) Tian, H. and Nonami, K., Robust Control of Flexible Rotor-Magnetic Bearing Systems Using Discrete Time Sliding Mode Control, JSME Int. J., Ser.C, Vol.37, No.3 (1994), pp.504-512.

( 6 ) Xu, Y. and Nonami, K., A Fuzzy Modeling of Active Magnetic Bearing System and Sliding Mode Control with Robust Hyperplane Using $\mu$-Synthesis Theory, JSME Int. J., Ser.C, Vol.46, No.2 (2003), pp.409415.

( 7 ) Utkin, V.I., Sliding Modes in Control and Optimization, (1992), Springer-Verlag.

( 8 ) Wang, F., Tsuda, K., Mizuno, H. and Nonami, K., Discrete Time Predictive Sliding Mode Control of Hot Strip Mill (Application of Linear Reaching Law), JSME Int. J., Ser.C, Vol.43, No.2 (2000), pp.293-299.

( 9 ) For example: Ogata, K., Modern Control Engineering, (1997), Prentice-Hall, Inc. 